\title{
Composição de ácidos graxos de margarinas à base de gordura hidrogenada ou interesterificada
}

\author{
Fatty acid composition of hydrogenated or interesterified margarines
}

Thaís Araújo CAVENDISH ${ }^{1 \star}$, Paula Bagno LEMOS², Renata Tiene YOKOTA², Tatiana França VASCONCELOS², Priscila Fernandes COÊLHO ${ }^{1}$, Marcelo BUZZI ${ }^{3}$, Marina Kiyomi ITO ${ }^{2,4}$

\begin{abstract}
Resumo
Foi analisado o conteúdo de ácidos graxos (AG) de 12 marcas de margarinas à base de óleos vegetais parcialmente hidrogenados ou interesterificados comercializados no Distrito Federal. As margarinas foram agrupadas pelo tipo de óleos utilizados em sua produção e o percentual médio de lipídios em GH-T (margarinas hidrogenadas com 50\% de lipídios), GH-L (hidrogenadas com 20\%), GI-T (interesterificadas com 65\%) e GI-L (interesterificadas com 30\%). O perfil de AG foi obtido por cromatografia gasosa em coluna capilar SP2560 ${ }^{\circ}$. O conteúdo de AG trans (AGT) no GH-T (7,91 $\pm 1,05$ g.100 g $\left.\mathrm{g}^{-1}\right)$ foi significativamente maior (p < 0,05) que no GH-L $(2,46 \pm 0,39$ g.100 g-1), GI-T $\left(1,29 \pm 0,47 \mathrm{~g} .100 \mathrm{~g}^{-1}\right)$ e GI-L $\left(0,65 \pm 0,24 \mathrm{~g} \cdot 100 \mathrm{~g}^{-1}\right)$. Houve diferença significativa no conteúdo total de AG saturados (AGS) e insaturados (AGI) entre os grupos GH-T e GI-T, sendo maior no GI-T, seguido de GH-T e sem diferença significativa entre GH-L e GI-L. Porém, as razões entre $\sum \mathrm{AGP} / \sum \mathrm{AGS}$ das margarinas não variou entre os grupos, enquanto as razões entre $\sum \mathrm{AGP} / \sum \mathrm{AGT}$ nas margarinas GI-T e GI-L foram superiores às demais. Os resultados obtidos indicam que as margarinas GI-T e GI-L seriam mais apropriadas para consumo humano, por possuírem menos AGT e uma melhor razão AGP/AGT que as demais.
\end{abstract}

Palavras-chave: ácidos graxos; isômeros trans; margarinas; hidrogenação; interesterificação; rotulagem.

\begin{abstract}
The content of fatty acids (FA) of 12 margarine brands produced with partially hydrogenated or interesterified vegetable oils marketed in Federal District was analyzed. The margarines were grouped into four groups by the type of oils used in their production and by the average percentage of lipids: GH-T (hydrogenated margarines with 50\% lipids), GH-L (hydrogenated with 20\%), GI-T (interesterified with $65 \%$ ) and GI-L (interesterified with $30 \%$ ). The fatty acid profile was obtained by gas chromatography in a SP2560 ${ }^{\circ}$ capillary column. The content of trans fatty acids (TFA) in GH-T $\left(7.91 \pm 1.05\right.$ g.100 g $\left.\mathrm{g}^{-1}\right)$ was significantly higher $(\mathrm{p}<0.05)$ than in GH-L $\left(2.46 \pm 0.39 \mathrm{~g} .100 \mathrm{~g}^{-1}\right)$, GI-T $\left(1.29 \pm 0.47 \mathrm{~g} .100 \mathrm{~g} \mathrm{~g}^{-1}\right)$ and GI-L $\left(0.65 \pm 0.24 \mathrm{~g} .100 \mathrm{~g}^{-1}\right)$. There was a significant difference in the total content of saturated fatty acids (SFA) and in the unsaturated fatty acids (UFA) among the groups GH-T and GI-T, being higher in GI-T, followed by GH-T and with no significant difference between GH-L and GI-L. The $\sum$ PUFA/ $\sum$ SFA ratios of the margarines were unchanged among the groups, while the $\Sigma$ PUFA/ $\Sigma$ TFA ratios in the GI-T and GI-L groups were higher than in the others. The results suggest that GI-L and GI-T margarines would be more appropriate for human intake due to lower content of TFA and higher PUFA/TFA ratio than the others.

Keywords: fatty acids; trans isomers; margarines; hydrogenation; interesterification; labeling.
\end{abstract}

\section{Introdução}

Estudos recentes indicam que os ácidos graxos trans (AGT) dietéticos têm efeitos adversos à saúde, aumentando o risco de desenvolvimento de doenças cardiovasculares (DCV), tais como a elevação da lipoproteína de baixa densidade (LDL) e a diminuição da lipoproteína de alta densidade (HDL), entre outros (ASCHERIO; WILLET, 1997; WILLET, 2006). A Organização Mundial de Saúde (OMS) preconiza que a ingestão diária de AGT seja inferior a $1 \%$ do consumo energético total diário (WHO, 2003). No Brasil, a Agência Nacional de Vigilância Sanitária (ANVISA) tornou obrigatória, em dezembro de 2003, a rotulagem nutricional com declaração do conteúdo de AGT. O prazo limite para adequação dos rótulos dos produtos foi dezembro de 2006 (BRASIL, 2006).
Os AGT estão presentes principalmente em alimentos processados que utilizam gordura vegetal parcialmente hidrogenada na sua formulação, tais como em margarinas, cremes vegetais, biscoitos, sorvetes, pães, batatas fritas de lanchonetes, produtos de pastelaria, bolos, massas, entre outros. Além dos alimentos processados, os produtos derivados de animal ruminante fornecem pequena quantidade de AGT, produzidos pela bio-hidrogenação (CHIARA; SICHIERI; CARVALHO, 2003).

O processo de hidrogenação de óleos foi desenvolvido com a função de modificar os óleos vegetais líquidos como substitutos da funcionalidade da gordura animal na produção

Recebido para publicação em 14/3/2008

Aceito para publicação em 20/6/2009 (003293)

${ }^{1}$ Programa de Iniciação Científica - PIC 2006/2007, 304 sul, bloco G, ap. 104, CEP 70337070, Asa Sul, Brasília - DF, E-mail: thaiscavendish@gmail.com

${ }^{2}$ Programa de Pós Graduação em Nutrição Humana, Universidade de Brasília - UnB, Campus Universitário Darcy Ribeiro, CEP 70910-900, Brasília - DF, Brasil

3 Rede SARAH de Hospitais de Reabilitação, Laboratório de Bioquímica Analítica

${ }^{4}$ Departamento de Nutrição, Universidade de Brasília - UnB, Campus Universitário Darcy Ribeiro, CEP 70910-900, Brasília - DF, Brasil

${ }^{*}$ A quem a correspondência deve ser enviada 
de alimentos. A margarina foi um dos produtos criados com o advento da hidrogenação, com o intuito de substituir a manteiga (VALENZUELA; MORGADO, 1999), considerada aterogênica. De acordo com a legislação brasileira atual, a margarina é o produto gorduroso em emulsão estável com leite ou seus constituintes ou derivados, com no máximo 95\% de gordura total (BRASIL, 1997). Assim, o conteúdo de AGT nas margarinas pode variar de acordo com o conteúdo e o grau de hidrogenação da matéria-prima gordurosa utilizada.

A margarina é um produto que compõe a dieta do brasileiro (MONDINI; MONTEIRO, 1995) e é um dos alimentos cujo conteúdo de AGT tem se adaptado à nova legislação. Uma alternativa interessante parece ser o uso de gorduras interesterificadas na sua formulação. $O$ processo de interesterificação possibilita a produção de gorduras livres ou com teor muito baixo de AGT, a partir do rearranjo dos ácidos graxos (AG) nas ligações éster do glicerol e consequente modificação do ponto de fusão e de cristalização da gordura (D'AGOSTINI, 2001), tendo como produto final uma matéria-prima com funcionalidades semelhantes, porém quimicamente diferentes das gorduras hidrogenadas. As gorduras interesterificadas são as novas alternativas para as hidrogenadas, porém há a preocupação em relação ao aumento no consumo de ácidos graxos saturados (AGS) a partir destes produtos (FARMANI; HAMEDI; SAFARI, 2008; LOPEZHERNANDEZ et al., 2007).

No Brasil, existe uma escassez de dados sobre AG em alimentos, especialmente dos AGT. Dessa forma, este trabalho teve como objetivos: 1) quantificar o teor de lipídios e o perfil de AG, com foco nos AGT, em margarinas comercializadas no Distrito Federal (DF); 2) compará-los com informações nutricionais dos rótulos e da Tabela Brasileira de Composição de Alimentos (TACO) (UNIVERSIDADE ESTADUAL DE CAMPINAS, 2006); e 3) identificar variações no conteúdo relativo de AGT entre diferentes marcas e grupos de margarinas.

\section{Material e métodos}

Foram analisadas 12 marcas de margarinas comercializadas no Distrito Federal. Estas marcas foram definidas a partir de informações de consumo alimentar obtidas de uma amostra selecionada aleatoriamente de duas regiões do Distrito Federal para investigar fatores de risco para o desenvolvimento de doenças crônicas não transmissíveis no DF, conforme detalhado em publicação anterior (YOKOTA et al., 2007). No questionário da pesquisa, foram perguntadas as marcas de margarinas normalmente consumidas, tendo sido relatadas 7 marcas. Outras 5 foram escolhidas em função da sua composição, completando as 12 marcas, que constituíram 4 grupos: $\mathrm{GH}-\mathrm{T}(\mathrm{n}=4)$, grupo composto por margarinas hidrogenadas tipo tradicional com percentual médio de lipídios de 50\%; GH-L ( $\mathrm{n}=2$ ), composto por margarinas hidrogenadas com percentual médio de lipídios de $20 \%$, considerado a versão light; GI-T $(n=4)$, composto por margarinas interesterificadas com percentual médio de lipídios de 65\%; e GI-L $(n=2)$, composto por margarinas interesterificadas com percentual médio de lipídios de 30\%. Para cada marca foram adquiridos 2 lotes, com exceção do grupo GI-L, totalizando 22 amostras analisadas em duplicatas. Todas as informações nutricionais dos rótulos das margarinas foram coletadas para posterior comparação.

As margarinas foram adquiridas em mercados locais e conservadas sob refrigeração para análise no Laboratório de Bioquímica da Nutrição da Universidade de Brasília (UnB). Os lipídios foram extraídos pelo método Folch (FOLCH; LEES; STANLEY, 1957) e os respectivos pesos aferidos por gravimetria. Os AG foram transmetilados em tolueno e solução metanólica de ácido sulfúrico a $1 \%$, à temperatura de $50{ }^{\circ} \mathrm{C}$ por 12 horas (HAMILTON, R. J.; HAMILTON, S. 1992). Os metil ésteres de AG foram ressuspendidos em isoctano a uma concentração de $10 \mathrm{mg} \cdot \mathrm{mL}^{-1}$, e $1 \mu \mathrm{L}$ foi injetado em coluna capilar SP 2560 (Supelco ${ }^{\circ}, 100 \mathrm{~m} \times 0,25 \mathrm{~mm} \times 0,20 \mu \mathrm{m}$ ) acoplada ao cromatógrafo a gás modelo GC 17 A (Shimadzu ${ }^{\circledR}$ ). As condições cromatográficas foram: temperatura do injetor a $250^{\circ} \mathrm{C}$; temperatura do detector a $250^{\circ} \mathrm{C}$; temperatura inicial da coluna de $125^{\circ} \mathrm{C}$ durante 3 minutos, aumento de $10^{\circ} \mathrm{C} /$ minuto até $170{ }^{\circ} \mathrm{C}, 3{ }^{\circ} \mathrm{C} /$ minuto até $176{ }^{\circ} \mathrm{C}, 2{ }^{\circ} \mathrm{C} /$ minuto até $185^{\circ} \mathrm{C}$, $1{ }^{\circ} \mathrm{C} /$ minuto até $190^{\circ} \mathrm{C}, 5^{\circ} \mathrm{C} /$ minuto até $240^{\circ} \mathrm{C}$ e temperatura final de $250^{\circ} \mathrm{C}$ (RATNAYAKE; HANSEN; KENNEDY, 2006). O gás de arraste foi o hidrogênio. Os resultados foram integralizados através do CBM101 e programa CLASS-GC10 (Shimadzu'); e a identificação dos AG foi feita com o auxílio de padrões comerciais externos (Sigma Aldrich ${ }^{\circledR}$ e AccuStandard ${ }^{\star}$ ).

Os AG foram calculados como percentuais em relação à área total de AG identificados e calculados para $100 \mathrm{~g}$ de margarina utilizando o fator de conversão de 0,956 (HOLLAND et al., 1997). Os valores obtidos de cada AG referem-se às médias das amostras em duplicatas. O programa estatístico SAS v. 8.2 foi utilizado para comparação dos resultados, aplicando-se análise de variância com $\mathrm{p}<0,05$ e teste Tukey para identificar as possíveis diferenças entre as amostras, além do teste $T$ de Student para identificar diferenças entre rótulos e análises.

\section{Resultados e discussão}

Foram identificados 27 AG (Tabela 1), sendo 3 deles os isômeros trans C18:1-9t, C18:1-11t, C18:2-9t,12c/9c,12t. Os AG encontrados em maior quantidade em todas as margarinas foram os ácidos linoleico (C18:2n-6) e o oleico (C18:1n-9), sendo a maior concentração de linoleico $\left(26,78 \pm 2,25 \mathrm{~g} \cdot 100 \mathrm{~g}^{-1}\right)$ encontrada na margarina GI-T. A maior concentração de ácido oleico (>13 g.100 g $\left.\mathrm{g}^{-1}\right)$ foi encontrada nas margarinas GH-T e GI-T. Dentre os ácidos graxos saturados, o palmítico (16:0) e o esteárico (18:0) foram os mais abundantes, principalmente nas margarinas do grupo GI-T, seguidos de GH-T. Entre os ácidos graxos trans, o elaídico (C18:1-9t) foi o mais abundante $\left(6,83 \pm 0,99 \mathrm{~g} \cdot 100 \mathrm{~g}^{-1}\right)$, presente nas margarinas do grupo GH-T e em menor quantidade no grupo GI-L $\left(0,16 \pm 0,13\right.$ g.100 g $\left.\mathrm{g}^{-1}\right)$. Os demais AG trans identificados nestas amostras foram em quantidades menores, porém significativamente maiores no grupo GH-T, em relação aos demais grupos. Os AG C20:1 e C18:3n-3 estão apresentados como valor único devido à sobreposição dos picos destes AG nos cromatogramas. Este valor foi considerado apenas para o cálculo do total de poliinsaturados. 
Tabela 1. Composição de ácidos graxos das margarinas à base de gordura vegetal hidrogenada ou interesterificada, Distrito Federal, 2007. Valores percentuais em $100 \mathrm{~g}$ de produto.

\begin{tabular}{|c|c|c|c|c|}
\hline & GH-T (\%) $(\mathrm{n}=4)$ & GH-L $(\%)(n=2)$ & GI-T (\%) $(\mathrm{n}=4)$ & GI-L $(\%)(n=2)$ \\
\hline Lipídios totais ácidos graxos & $51,08 \pm 3,93$ & $20,06 \pm 1,58$ & $63,08 \pm 5,75$ & $28,82 \pm 2,66$ \\
\hline $\mathrm{C} 4: 0$ & $0,04 \pm 0,03$ & $0,02 \pm 0,00$ & $0,04 \pm 0,03$ & $0,00 \pm 0,00$ \\
\hline C6:0 & $0,01 \pm 0,01$ & $0,00 \pm 0,00$ & $0,01 \pm 0,01$ & $0,00 \pm 0,00$ \\
\hline C10:0 & $0,02 \pm 0,01^{\mathrm{a}}$ & $0,00 \pm 0,00^{\mathrm{b}}$ & $0,02 \pm 0,01^{\mathrm{a}}$ & $0,01 \pm 0,00^{\mathrm{a}}$ \\
\hline C12:0 & $0,12 \pm 0,13^{\mathrm{a}}$ & $0,02 \pm 0,02^{\mathrm{a}}$ & $1,04 \pm 0,35^{\mathrm{b}}$ & $0,22 \pm 0,26^{\mathrm{a}}$ \\
\hline C14:0 & $0,11 \pm 0,07^{\mathrm{a}}$ & $0,02 \pm 0,01^{\mathrm{a}}$ & $0,71 \pm 0,22^{\mathrm{b}}$ & $0,18 \pm 0,15^{\mathrm{a}}$ \\
\hline $\mathrm{C} 17: 0$ & $0,05 \pm 0,01^{\mathrm{a}}$ & $0,02 \pm 0,00^{\mathrm{b}}$ & $0,06 \pm 0,01^{\mathrm{a}}$ & $0,02 \pm 0,00^{\mathrm{b}}$ \\
\hline C18:0 & $5,13 \pm 1,13^{a}$ & $2,01 \pm 0,11^{\mathrm{b}}$ & $6,26 \pm 0,92^{\circ}$ & $2,42 \pm 1,22^{b}$ \\
\hline C20:0 & $0,29 \pm 0,04^{\mathrm{a}}$ & $0,15 \pm 0,02^{\mathrm{b}}$ & $0,33 \pm 0,04^{a}$ & $0,15 \pm 0,05^{b}$ \\
\hline C21:0 & $0,02 \pm 0,00^{\mathrm{a}}$ & $0,01 \pm 0,00^{\mathrm{b}}$ & $0,02 \pm 0,00^{\mathrm{a}}$ & $0,01 \pm 0,00^{\mathrm{b}}$ \\
\hline C22:0 & $0,36 \pm 0,09^{a}$ & $0,31 \pm 0,06^{\mathrm{a}}$ & $0,37 \pm 0,06^{\mathrm{a}}$ & $0,14 \pm 0,06^{b}$ \\
\hline $\mathrm{C} 16: 1 \mathrm{n}-7$ & $0,05 \pm 0,01^{\mathrm{a}}$ & $0,02 \pm 0,01^{\mathrm{b}}$ & $0,05 \pm 0,01^{\circ}$ & $0,03 \pm 0,01^{\mathrm{a}, \mathrm{b}}$ \\
\hline $\mathrm{C} 17: 1$ & $0,01 \pm 0,01^{\mathrm{a}}$ & $0,01 \pm 0,00^{\mathrm{a}}$ & $0,02 \pm 0,00^{\mathrm{b}}$ & $0,01 \pm 0,00^{\mathrm{a}, \mathrm{b}}$ \\
\hline C18:1n-9 & $13,50 \pm 1,73^{\mathrm{a}}$ & $4,80 \pm 0,16^{\mathrm{b}}$ & $13,43 \pm 2,02^{a}$ & $9,62 \pm 4,62^{\mathrm{a}, \mathrm{b}}$ \\
\hline C22:1n-9 & $0,01 \pm 0,01$ & $0,01 \pm 0,00$ & $0,01 \pm 0,01$ & $0,00 \pm 0,00$ \\
\hline C24:1 & $0,01 \pm 0,01$ & $0,01 \pm 0,01$ & $0,01 \pm 0,01$ & $0,00 \pm 0,00$ \\
\hline Total de AGM & $13,58 \pm 1,74^{\mathrm{a}}$ & $4,85 \pm 0,17^{\mathrm{b}}$ & $13,53 \pm 2,02^{\mathrm{a}}$ & $9,66 \pm 4,30^{\mathrm{a}, \mathrm{b}}$ \\
\hline C18:2n-6 & $14,35 \pm 2,71^{\mathrm{a}}$ & $6,57 \pm 0,16^{b}$ & $26,78 \pm 2,25^{c}$ & $9,95 \pm 1,91^{\mathrm{a}, \mathrm{b}}$ \\
\hline C18:3n-6 & $0,17 \pm 0,07^{\mathrm{a}}$ & $0,07 \pm 0,02^{\mathrm{b}}$ & $0,26 \pm 0,11^{\circ}$ & $0,10 \pm 0,09^{\mathrm{a}, \mathrm{b}}$ \\
\hline $\mathrm{C} 20: 1+\mathrm{C} 18: 3 n-3$ & $1,31 \pm 0,25^{\mathrm{a}}$ & $0,66 \pm 0,08^{b}$ & $2,68 \pm 0,17^{c}$ & $1,36 \pm 0,61^{\circ}$ \\
\hline C20:2 & $0,01 \pm 0,01^{\mathrm{a}}$ & $0,00 \pm 0,00^{\mathrm{a}}$ & $0,02 \pm 0,00^{\mathrm{b}}$ & $0,00 \pm 0,00^{\mathrm{a}}$ \\
\hline Razão $\Sigma$ AGP/ $\Sigma$ AGS & 1,51 & 1,84 & 1,92 & 1,62 \\
\hline
\end{tabular}

Os valores seguidos por letras diferentes na mesma linha são significativamente diferentes $(\mathrm{p}<0,05)$ pelo teste Tukey.

Na TACO (UNIVERSIDADE ESTADUAL DE CAMPINAS, 2006), que é a primeira tabela brasileira com informações sobre AGT em alimentos nacionais, as margarinas que utilizam 65\% de óleos vegetais parcialmente hidrogenados na sua composição apresentam predominância dos AG linoleico (C18:2n-6; 19,48\%), oleico (C18:1n-9; 17,87\%), C18:1-t (8,69\%), palmítico $(16: 0 ; 8,29 \%)$ e esteárico $(18: 0 ; 5,75 \%)$. As margarinas que empregam óleos vegetais interesterificados apresentam um conteúdo reduzido de C18:1-t (0,12\%), e aumentados em relação aos linoleico e palmítico das hidrogenadas (UNIVERSIDADE ESTADUAL DE CAMPINAS, 2006). Os resultados do presente estudo são comparáveis aos da tabela TACO, havendo ligeira diferença no percentual de alguns AG. Em relação aos AGT, o resultado da margarina GH-T $\left(7,91 \mathrm{~g} .100 \mathrm{~g}^{-1}\right)$ é próximo ao da tabela TACO $\left(8,69\right.$ g.100 g $\left.{ }^{-1}\right)$. Entretanto, em relação às margarinas GI-T, os valores são diferentes (TACO: 0,12 g.100 g ${ }^{-1}$ e neste estudo: $1,29 \mathrm{~g} .100 \mathrm{~g}^{-1}$ ). Uma possível explicação é o fato de a tabela TACO ter identificado apenas um AGT, além das diferenças prováveis de marcas e de composição entre as margarinas deste estudo com as analisadas na TACO. Observase ainda, tanto na tabela TACO como no presente estudo, que nas margarinas GI, o reduzido teor de AGT foi compensado pela maior quantidade de AG insaturados (C18:1n-9 no GI-L e C18:2n-6 no GI-T) e saturados (16:0).

Analisando os teores dos ácidos graxos por grau de saturação (Tabela 1), verifica-se que as margarinas GI-T apresentam maior conteúdo de AGS (16,56 $\pm 0,80$ g.100 g $\left.\mathrm{g}^{-1}\right)$ bem como de ácidos graxos monoinsaturados (AGM) + ácidos graxos poli-insaturados (AGP) $\left(45,37 \pm 8,84 \mathrm{~g} .100 \mathrm{~g}^{-1}\right)$, em relação aos outros grupos. Este resultado é relevante, uma vez que há a 
preocupação do aumento significativo do conteúdo de gorduras saturadas em consequência da redução de gorduras trans nos produtos alimentícios, pois as primeiras também contribuem para o aumento do risco de DCV (GRUNDY; DENKE, 1990). Contudo, as razões $\sum \mathrm{AGP} / \Sigma \mathrm{AGS}$ das margarinas não variaram entre os grupos (Tabela 1). Outro resultado interessante é o fato de as razões $\sum$ AGP/ $\sum$ AGT nas margarinas GI-T e GI-L terem sido superiores às demais.

As quantidades de trans nas marcas analisadas não variaram dentro de cada um dos quatro grupos. Entretanto, nas margarinas do grupo GH-T, houve variação entre as marcas, tanto para os AGS $(\mathrm{p}<0,01)$ como para os AGP $(\mathrm{p}<0,01)$.

Comparando-se os valores de AG descritos nos rótulos das margarinas com os obtidos nas análises laboratoriais, observouse que, para os AGS, os rótulos mostraram valores próximos, porém maiores $(\mathrm{p}<0,05)$ para as margarinas tradicionais. Para os AGI, os rótulos das margarinas tradicionais e GH-L também foram maiores $(\mathrm{p}<0,05)$. Em relação aos AGT (Figura 1), o
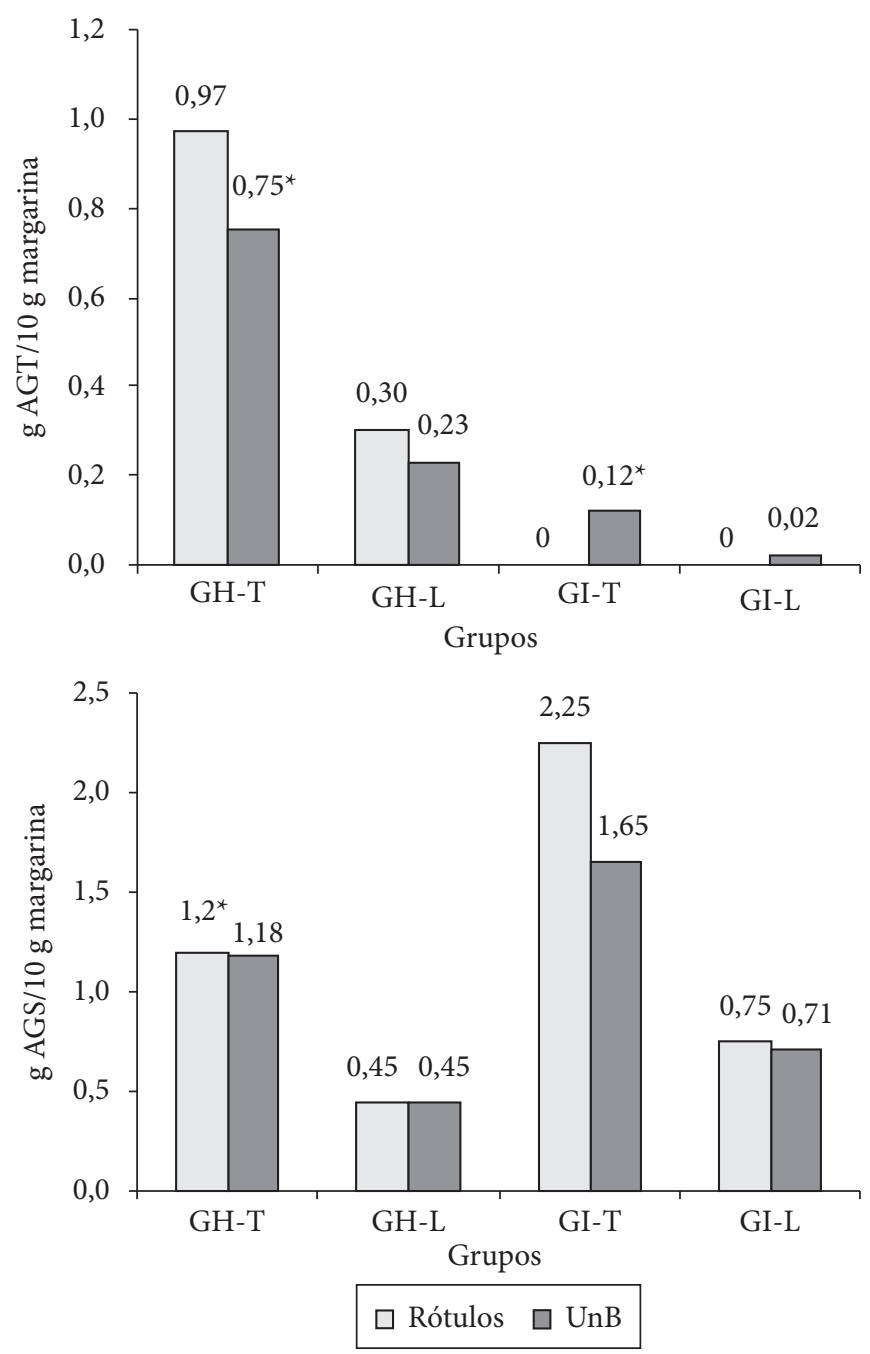

Figura 1. Teores de AGT e AGS apresentados nos rótulos das margarinas e nas análises laboratoriais (g/porção de $10 \mathrm{~g}) .{ }^{*} \mathrm{p}<0,05$ pelo teste $T$ de Student. rótulo do grupo GH-T foi maior e o do grupo GI-T foi menor do que nas análises $(p<0,05)$. Ressalta-se que, apesar destas diferenças, todos os valores de AGT dos rótulos estavam dentro dos parâmetros previstos pela legislação (BRASIL, 2006).

Wagner, Auer e Elmadfa (2000) detectaram 1,6\% de AGT em relação ao total de lipídios das margarinas analisadas. Torres, Casal e Oliveira (2002), em estudo realizado em Portugal, encontraram o valor médio de 2,5\% de AGT nas margarinas, assim como Larqué et al. (2003), em margarinas espanholas. No estudo de Triantafillou, Zaografos e Katsikas (2003), o conteúdo de AGT de margarinas gregas foi considerado relativamente baixo, porém, segundo os autores, havia uma quantidade elevada de AGS, não condizente com os rótulos. Huang et al. (2006), analisando diversos alimentos consumidos em comunidades afroamericanas, constataram que as margarinas apresentavam um teor de 19,13\% de AGT. No presente estudo, o conteúdo de AGT de margarinas do grupo GH-T é intermediário em relação aos estudos mencionados. No nosso estudo, a diferença no conteúdo de $\mathrm{AG}$ dos rótulos em relação à análise pode ser decorrente de diferenças nos métodos de análise.

Em relação aos óleos utilizados, as marcas apenas informavam se eram hidrogenados e/ou interesterificados, sem especificar o tipo e/ou os tipos de óleos contidos nestas misturas. Além disso, algumas marcas apenas relatavam "óleos vegetais" entre os ingredientes, sem especificar o processamento aplicado. Apesar de não ser informação obrigatória em rótulo, a declaração dos tipos de óleos utilizados é de interesse para o consumidor, pois uma quantidade inferior a $0,2 \mathrm{~g}$ de AGT por porção pode ser legalmente considerada nula (BRASIL, 2006), mas pode ser significante quando a ingestão é maior do que uma porção.

\section{Conclusões}

Conclui-se que os rótulos das margarinas analisadas neste estudo estão adequados quanto à legislação em vigor. A comparação entre os teores de AG entre as margarinas interesterificadas e hidrogenadas indica que as interesterificadas seriam mais recomendadas para consumo humano, por possuírem menor teor de AGT que as demais, especialmente GI-L. Soma-se ainda o fato de as razões entre $\sum$ AGP/ $\sum$ AGT nestas margarinas terem sido superiores às demais, resultando em maior fornecimento de AGP aos consumidores.

\section{Agradecimentos}

Ao Conselho Nacional de Desenvolvimento Científico e Tecnológico - CNPq pelo auxílio financeiro e à Coordenação de Aperfeiçoamento de Pessoal de Nível Superior - CAPES pela bolsa.

\section{Referências bibliográficas}

ASCHERIO, A.; WILLET, W. C. Health effects of trans fatty acids. American Journal of Clinical Nutrition, v. 66, n. 4, p. 1006-10, 1997.

BRASIL. Ministério da Agricultura e do Abastecimento. Portaria n. 372, de 04 de setembro de 1997. Regulamento técnico de identidade e 
qualidade de margarina. Diário Oficial da União, Poder Executivo, Brasília, DF, 08 set. 1997, Seção 1, p. 19702.

BRASIL. Agência Nacional de Vigilância Sanitária. Resolução RDC n. 360, de 26 de dezembro de 2003. Regulamento técnico sobre rotulagem nutricional de alimentos embalados, tornando obrigatória a rotulagem nutricional. Diário Oficial da União, Poder Executivo, Brasília, DF, 26 dez. 2006.

CHIARA, V. L.; SICHIERI, R.; CARVALHO, T. D. S. F. D. Teores de ácidos graxos trans de alguns alimentos consumidos no Rio de Janeiro. Revista de Nutrição, v. 16, n. 2, p. 227-233, 2003.

D'AGOSTINI, D. Obtenção de lipídios estruturados por interesterificação de triacilgliceróis de cadeia media e longa. São Paulo, 2001. 167 p. Tese (Doutorado em Tecnologia de Alimentos) Universidade de São Paulo - USP.

FARMANI, J.; HAMEDI, M.; SAFARI, M. Production of zero trans Iranian vanaspati using chemical transesterification and blending techniques from palm olein, rapeseed and sunflower oils. International Journal of Food Science \& Technology, v. 43, n. 3, p. 393-399, 2008.

FOLCH, J.; LEES, M.; STANLEY, G. H. S. A simple method for the isolation and purification of total lipids from animal tissues. The Journal of Biological Chemistry, v. 226, n. 1, p. 497-509, 1957.

HAMILTON, R. J.; HAMILTON, S. Lipid analysis: a practical approach. IRL Press, 1992.

HOLLAND, B. et al. McCance and Widdowson's: the composition of foods. 5 ed.Cambridge: The Royal Society of Chemistry, 1997.

HUANG, Z. et al. Trans fatty acid content of selected foods in an African-American Community. Journal of Food Science, v. 71, n. 6, p. C322-C327, 2006.

LARQUÉ, E. et al. Composición en ácidos grasos de las margarinas de mayor consumo en España y su importancia nutricional. Grasas y Aceites, v. 54, n. 1, p. 65-70, 2003.

LOPEZ-HERNANDEZ, A. et al. Interesterification of sesame oil and a fully hidrogenated fat using an immobilized lipase catalyst in both batch and continuous-flow processes. European Journal of Lipid Science and Technology, v. 109, n. 12, p. 1147-1159, 2007.

MONDINI, L.; MONTEIRO, C. A. Mudanças no padrão de alimentação. In: MONTEIRO, C. A. (org.). Velhos e novos males da saúde no Brasil: a evolução do país e de suas doenças. São Paulo: Editora Hucitec: 1995. p. 79-89.
RATNAYAKE, W. M. N.; HANSEN, S. L.; KENNEDY, M. P. Evaluation of the CP-Sil88 and SP-2560 GC columns used in the recently approved AOCS Official Method Ce 1h-05: determination of cis-, trans-, saturated, monounsaturated and polyunsaturated fatty acids in vegetable or non-ruminant animal oils and fats by capillary GLC method. Journal of the American Oil Chemists' Society, v. 83, n. 6, p. 475-488, 2006.

GRUNDY, S. M.; DENKE, M. A. Dietary influences on serum lipids and lipoproteins. J. Lip. Research, v. 31, n. 7, p. 1149-1172, 1990.

TORRES, D.; CASAL, S.; OLIVEIRA, B. Fatty acid composition of Portuguese spreadable fats with emphasis on trans isomers. European Food Research and Technology, v. 21, n. 4, p. 108-11, 2002.

TRIANTAFILLOU, D.; ZOGRAFOS, V.; KATSIKAS, H. Fatty acid content of margarines in the Greek market (including trans fatty acids): a contribution to improving consumers' information. International Journal of Food Science \& Technology, v. 54, n. 2, p. 135-41, 2003.

UNIVERSIDADE ESTADUAL DE CAMPINAS - UNICAMP. Tabela brasileira de composição de alimentos. 2 ed. São Paulo: Núcleo de Estudos e Pesquisas em Alimentação, 2006. (versão 2) Disponível em:<http://www.unicamp.br/nepa/taco/>.

VALENZUELA, A.; MORGADO, N. Trans fatty acid isomers in human health and in the food industry. Biological Research, v. 32, n. 4, p. 273-87, 1999 .

WAGNER, K.; AUER, E.; ELMADFA, I. Content of trans fatty acids in margarines, plant oils, fried products and chocolate spreads in Austria. European Food Research and Technology, v. 210, n. 4, p. 237-41, 2000 .

WILLETT, W. C. Trans fatty acids and cardiovascular disease: epidemiological data. Atherosclerosis Supplements, v. 7, n. 2, p. 5-8, 2006.

WORLD HEALTH ORGANIZATION - WHO. Diet, nutrition and the prevention of chronic diseases. Geneva, 2003. (Tecnical Report Series 916).

YOKOTA, R. T. et al. Prevalência de fatores de risco para doenças crônicas não-transmissíveis em duas regiões do Distrito Federal. Comunicação em Ciências da Saúde, v. 18, n. 4, p. 289-296, 2007. 